\title{
Introduction to the third issue of World Nutrition for 2020
}

Occasionally in life, we have the experience of seeing a photo of a beloved person no longer with us and feel, sadly jolted, the finality of death. This has happened to me several times preparing this issue, which includes memorials to two of the leading international practitioners in our field, Arne Oshaug of Norway and Björn Ljungqvist of Sweden. As we have done with other giants in public health nutrition in the past, we include both a longer piece about their lives from people close to them and tributes to them from numerous colleagues.

This issue includes six papers from Africa. Among papers presenting original research, from Zimbabwe, Moyo et al. do a barrier analysis to examine factors linked to successful exclusive breastfeeding; Ncube Murakwani et al. look at whether participation in Care Groups was associated with increased dietary diversity in women and children. From Nigeria, theme et al. explore the damage to diets that the COVID-19 pandemic there has done. Tsrah et al. analyze advertising provided to customers at the major malls in Accra, Ghana, finding that it is almost exclusively unhealthy foods that are promoted. Then, in a literature review, Otekunrin et al. examine the progress and challenges of three decades of fighting hunger in Africa. Finally, Moyo and Thow provide a commentary on three different ways of looking at the human right to food and nutrition in South Africa.

From Turkey, Muftuoglu and Bayram measure the relationship between nutrition habits, social physique anxiety and physical activity levels of adolescent students. From the USA, Rhodes looks at how import refusals for pathogen violations respond to a recession.

Relevant to World Nutrition recently publishing papers on Care Groups, Shrimpton provides a commentary reviewing them and other community-based approaches for improved maternal and child survival and development. In a research letter, Maximiano et al. provide data on iron deficiency anemia in a sample of Portuguese infants. Finally, in his Good Questions editorial for this issue, George Kent expresses concern about the promotion of a new technology for making infant formula, Biomilq, which is falsely claiming to be making "cultured human breast milk.”

--Ted Greiner, Editor-in-Chief, World Nutrition 